\title{
DETERMINACIÓN DE FACTORES RELACIONADOS CON EL ABANDONO DE LA PRÁCTICA DEPORTIVA EN EX NADADORES COSTARRICENSES
}

\author{
Fabián Víquez Ulate ${ }^{1}$ y Gerardo Araya Vargas ${ }^{2}$ \\ ${ }^{1}$ Universidad Nacional, favz13@ gmail.com \\ ${ }^{2}$ Universidad de Costa Rica, aliuncepa@yahoo.com
}

\section{RESUMEN}

\begin{abstract}
El propósito de este estudio fue determinar la importancia de distintas dimensiones, como posibles causas que permiten explicar el abandono de la práctica deportiva de la natación, en ex nadadores costarricenses. Sujetos: participaron 51 ex nadadores, 17 hombres ( $22 \pm 3.4$ años) y 34 mujeres (22,5 \pm 3.4 años), de distintos equipos de natación de Costa Rica. Se les aplicó el Cuestionario de Causas de Abandono de la Práctica Deportiva (Gould et al, 1982; citado por Salguero, Tuero y Márquez, 2003). Resultados: se obtuvo una relación positiva entre las percepciones de escasa habilidad y de bajo rendimiento $(r: 0,372)$, y las relaciones negativas entre años de práctica y escasa habilidad ( $r$ :-0,337), también entre años de práctica e influencia de otros $(r:-0,286)$, y entre años de práctica y edad de comienzo en la natación ( $r$ :0,622). Hubo interacción significativa de las dimensiones por género de los participantes $(F: 2,952$; $p<0,05)$ y de dimensiones por edad de inicio de la práctica de la natación $(F: 3,147 ; p<0,01)$. Se encontró interacción entre dimensiones y deseo de volver a practicar la natación ( $F: 2,485 ; p>0,05)$. También diferencias significativas en los años de práctica, donde los sujetos que iniciaron antes de los 10 años de edad, tuvieron un promedio de años de práctica más alto que los sujetos que empezaron después de los 10 años de edad $(F: 21,465 ; p<0,05)$. Se encontró interacción significativa de dimensiones por horas de práctica semanal de natación ( $F$ : 2,072; $p<0,05)$. No hubo diferencias significativas $(F: 2,535 ; p>0,05)$, según la cantidad de horas de entrenamiento semanal que tenían los sujetos siento nadadores activos. Conclusiones: como posibles causas de abandono, para hombres, fueron la falta de diversión y la baja forma; en mujeres fue la escasa atmósfera de equipo. En el grupo que inició después de los 10 años de edad valoran con una importancia similar las dimensiones como posibles causas de retiro, mientras que el grupo que inició la natación antes de los 10 años de edad, la escasa atmósfera de equipo, la baja forma y la falta de diversión, son las posibles causas de abandono, más importantes. En los sujetos que sí deseaban volver a nadar, se mostró que la escasa atmósfera de equipo, la baja forma y la escasa habilidad fueron las dimensiones más importantes como posibles causas de abandono. En el caso de los sujetos que no desean volver a nadar, la falta de diversión fue la causa más importante. En el grupo de sujetos que nadaban menos de seis horas, la escasa atmósfera de equipo es la causa más importante. Para los sujetos que nadaban de 6 a 12 horas, la causa más importante fue la dimensión, falta de diversión. Asimismo se observó que en la cercanía del cambio del colegio a la universidad se dan los porcentajes más altos de abandono.
\end{abstract}

\section{DETERMINATION OF FACTORS RELATED TO THE ABANDONMENT OF THE PRACTICE OF SWIMMING BY FORMER COSTA RICAN SWIMMERS}

\begin{abstract}
The objective of this study was to determine the importance of different dimensions as the possible causes for the abandonment of the practice of swimming by former Costa Rican swimmers. Subjects: 51 former swimmers participated, including 17 men (22 \pm 3.4 years of age) and 34 women ( $22.5 \pm 3.4$ years of age) from different Costa Rican swimming teams. The Questionnaire of Reasons for Attrition in the Practice of Sports (Gould et al, 1982; cited by Salguero, Tuero, and Márquez, 2003) was used. Results: A positive relationship was noted between perceptions of low skill and low performance ( $r$ : 0.372), while negative relationships were noted between years of practice and low skill ( $r:-0.337)$, years of practice and influence from others ( $r:-0.286)$, and years of practice and starting age for swimming $(r:-0.622)$. A significant interaction was found in the dimensions gender of participants $(F: 2.952 ; p<0.05)$ and starting age for swimming $(F: 3.147 ; p<0.01)$. An interaction was also found between dimensions and the desire to swim again $(F: 2.485 ; p>0.05)$. There were also significant differences in the years of practice; that is, subjects who started before the age of 10 had a higher average of years of practice than those who started later ( $F: 21.465 ; p<0.05)$. Additionally, a significant interaction was found in weekly hours of swimming practice $(F: 2.072 ; p<0.05)$. There were no significant
\end{abstract}




\section{MHSalud}

URL www.una.ac.cr/mhsalud

differences (F: 2.535; p>0.05) according to weekly hours of training when subjects were actively swimming. Conclusions: Possible reasons for dropping out were lack of enjoyment and poor physical condition for men and perception of lack of team spirit for women. The group that started after the age of ten gives similar importance to all the dimensions as possible reasons for dropping out, while the group that began swimming before the age of ten sees lack of team spirit, lack of enjoyment, and poor physical condition as the key reasons for dropping out. The most important dimensions that represented the reasons for abandonment in subjects who wanted to swim again were lack of team spirit, poor physical condition and low skill, while lack of enjoyment was the most important reason for subjects who do not want to swim again. In the group of subjects who would swim less than six hours, lack of team spirit is the most important cause, while the dimension lack of enjoyment was the most important reason for subjects who would swim 6 to 12 hours. Additionally, the highest dropout percentages were observed in the transition from secondary school to college.

KEY WORDS: abandonment, swimming and gender.

\section{INTRODUCCIÓN}

Los jóvenes deportistas entran a practicar deporte por varios motivos: divertirse, satisfacción personal, competir, estatus, salud, aprender destrezas, recreación, amigos, entre otros. Continuarán en el deporte motivado por diversas causas, pero lo abandonarán pronto si no son satisfechas sus necesidades (Águila y Andujar, 2000; Salguero, Tuero y Márquez, 2003 y Valdés, 1996).

El abandono deportivo no se produce por un factor aislado, sino que existe una relación entre varios. Pero no se puede suponer que los deportistas que interrumpen la asistencia a un deporte nunca lo retomarán, pues investigaciones muestran que en deportistas se puede distinguir niveles de abandono, como aquellos que lo hacen para unirse a otro. Un ejemplo fue un estudio hecho por Gould y otros (1982 citado por Trepode, 2001) donde el $80 \%$ de los nadadores que habían abandonado el deporte, se habían reintegrado o planeaban retornar al mundo deportivo. En otro estudio, realizado por Klint y Weiss (1986, citado por Trepode, 2001) hallaron que 35 de 37 desertores, se reintegraron a otros deportes, considerando que el abandono deportivo es un proceso continuo que va desde que deportista se retira, que pasa a practicar otro o el mismo a diferente intensidad, hasta el abandono definitivo (Salguero, Tuero y Márquez, 2003 y Trepode, 2001).

Estudios han tratado de investigar que motiva a los deportistas a esta decisión. Un estudio hecho por García (2001), encontró motivos de abandono como: salía cansado del trabajo (33\%), por pereza $(32 \%)$, estudios (32\%), falta de tiempo (17\%), instalaciones inadecuadas (15\%), instalaciones deportivas muy lejanas (14\%), falta de apoyo y estímulo (14\%) y una última razón: los amigos no hacían deporte (10\%). Otro estudio realizado por Salguero, Tuero y Márquez (2003), cita motivos de abandono en nadadores como: bajo rendimiento, falta de diversión, escasa habilidad, influencia de otros, baja forma/interés y falta de atmósfera de equipo (Salguero, Tuero y Márquez, 2003 y Ruiz y García, 2002).

Por otro lado, Ruiz y García (2002), notaron que el abandonado de la práctica deportiva corresponde frecuentemente a las poblaciones cercanas a épocas de cambios de estudios obligatorios a opcionales, o al mundo laboral o al familiar, entre otros, coincide con la ruptura de los grupos primarios de relación de la primera etapa de juventud; esto viene a indicar que a medida que se va avanzando en los niveles educativos, el abandono que se produce es mayor por la cantidad de actividades diferentes al deporte que se deben realizar.

Como se ha mencionado la natación no escapa del abandono, pues una situación importante particular en este deporte es el sobreentrenamiento infantil, con el objetivo de lograr campeones a 


\section{MHSalud}

URL www.una.ac.cr/mhsalud

temprana edad. En la actualidad se ha comprobado que la edad del máximo rendimiento en hombres está por encima de los 21 años, siendo más alta para los nadadores velocistas; mientras que las mujeres llegan un poco antes, debido a que el desarrollo fisiológico es anterior al de los hombres. También es sabido que una edad adecuada para el inicio deportivo ronda los 8 años, dando un espacio de trabajo de más de diez años, es decir los niños y niñas alcanzan rendimientos máximos luego de 6 a 10 años de entrenamiento (Águila y Andujar, 2000 y Año, 1997).

Otro factor que puede influir en el abandono son los padres que desean que sus niños sean destacados en el deporte. Asimismo, si en la familia, existió algún deportista ya la experiencia previa puede afectar positivamente en la adherencia al deporte. Los compañeros de equipo y amigos pueden ser otro factor influyente pues como se ha tratado, a menor edad, la influencia de la familia es más fuerte, pero después de los siete años esta empieza a decaer cobrando terreno el grupo de amigos hasta llegar a invalidar casi por completo la influencia familiar; el grupo de amigos puede influir tanto en forma negativa como positiva. Otros factores pueden ser horarios, distancia del lugar de entrenamiento, cohesión grupal, entre otros (Weinberg y Gould, 1996 y Año, 1997).

Por tanto, el propósito de este estudio fue determinar la importancia de distintas dimensiones como posibles causas que permiten explicar el abandono de la práctica deportiva de la natación, en exnadadores costarricenses.

\section{METODOLOGÍA}

Sujetos: En este estudio participaron 51 exnadadores, 17 hombres con edad de $22 \pm 3.4$ años y 34 mujeres con edad promedio de $22.5 \pm 3.4$ años, pertenecientes a siete equipos de natación de Costa Rica de distintas localidades. De estos, 23 nadadores alcanzaron un nivel competitivo nacional y los 28 restantes un nivel internacional, por lo menos una temporada.

Instrumentos: Se utilizó el Cuestionario de Causas de Abandono de la Práctica Deportiva elaborado por Gould y otros (1982, citado por Salguero, Tuero y Márquez, 2003) y validado en España por Salguero, Tuero y Márquez (2003).

El Cuestionario de Causas de Abandono de la Práctica Deportiva está conformado por 29 ítems, pertenecientes a seis dimensiones: "Bajo Rendimiento", "Falta de diversión", "Escasa habilidad", "Influencia de otros", "Baja forma" y "Escasa atmósfera de equipo".

Los coeficientes alfa de Cronbach de las diferentes escalas alcanzaron o se aproximaron al nivel mínimo de aceptación Nunnally (1978) e indicaron una consistencia interna apropiada para las distintas dimensiones. De acuerdo al análisis para "Bajo Rendimiento" 0.77, "Falta de diversión" 0.76, "Escasa habilidad" 0.71, "Influencia de otros" 0.58, "Baja forma/ interés" 0.72 y "Falta de Atmósfera de Equipo" 0.87 (Salguero, Tuero y Márquez, 2003).

Procedimientos: Se buscó la ayuda de entrenadores, se consiguieron direcciones de habitación y números telefónicos de algunos, se asistió a eventos competitivos de natación para aficionados todo con el objetivo de encontrar excompetidores. En caso de acceder a colaborar voluntariamente se llevó a cabo la administración del cuestionario. Los sujetos debieron haber practicado a nivel competitivo por lo menos una temporada completa. Las instrucciones para llenar el cuestionario se anexaron por escrito y se estableció un tiempo aproximado de 15 minutos para completar el instrumento 


\section{MHSalud}

URL www.una.ac.cr/mhsalud

Análisis estadístico: Se aplicó estadística descriptiva del cálculo de promedio y desviaciones estándar para edad, años de práctica, equipo, género y dimensiones del Cuestionario de Causas de Abandono de la Práctica Deportiva. Además se aplicó como estadística inferencial correlaciones de Pearson para relacionar los años de práctica con distintas variables medidas en el estudio. Se aplicó un ANOVA de 3 vías para comparar las seis dimensiones del Cuestionario de Causas de Abandono de la Práctica Deportiva, aplicado a los sujetos, en función de género y de su edad de inicio de práctica (antes y después los 10 años). Por otro lado, se realizó un ANOVA de 3 vías para comparar las 6 dimensiones del Cuestionario de Causas de Abandono de la Práctica Deportiva, aplicado a los sujetos, en función de género y su deseo de volver a practicar natación competitiva. También se aplicó un Análisis de Varianza de un vía para determinar si existía diferencia en los años de práctica que tuvieron los sujetos según la cantidad de horas de entrenamiento semanal que tenían.

Por su parte se aplicó Eta cuadrado cuando fue necesario y análisis de efectos post-hoc simples cuando lo ameritaba.

Se aplicó análisis de interacciones simples, de aspectos simples post-hoc de Tukey y LSD, cuando fue necesario y una prueba de Chi cuadrado para determinar diferencias en cuanto al deseo de volver a la práctica de natación. El procesamiento de la información fue mediante el Paquete Estadístico para Ciencias Sociales (S.P.S.S.) versión 8.0 para Windows

\section{RESULTADOS}

A continuación se presentan los resultados de este estudio.

Tabla 1. Promedios y desviaciones estándar de la edad (años), edad de comienzo y de finalización (años) y años de práctica según equipo y género

\begin{tabular}{cccc}
\hline & $\begin{array}{c}\text { Hombres } \\
(\mathbf{n = 1 7})\end{array}$ & $\begin{array}{c}\text { Mujeres } \\
(\mathbf{n = 3 4})\end{array}$ & $\begin{array}{c}\text { Total } \\
(\mathbf{n = 5 1})\end{array}$ \\
\hline Edad & $22,00 \pm 3,27$ & $22,55 \pm 3,43$ & $22,37 \pm 3,35$ \\
Edad de comienzo en la natación & $9,88 \pm 2,82$ & $9,29 \pm 2,86$ & $9,49 \pm 2,83$ \\
Edad de finalización en la natación & $16,35 \pm 2,42$ & $16,97 \pm 3,04$ & $16,76 \pm 2,83$ \\
Años de práctica & $6,47 \pm 3,18$ & $7,67 \pm 3,67$ & $7,27 \pm 3,53$ \\
\hline
\end{tabular}

Tabla 2. Correlaciones de Pearson de los años de práctica con distintas variables medidas 


\section{MHSalud}

URL www.una.ac.cr/mhsalud

\begin{tabular}{|c|c|c|c|c|c|c|c|c|c|}
\hline & $\begin{array}{c}\text { Bajo } \\
\text { rendimiento }\end{array}$ & $\begin{array}{c}\text { Falta de } \\
\text { diversión }\end{array}$ & $\begin{array}{c}\text { Escasa } \\
\text { habilidad }\end{array}$ & $\begin{array}{c}\text { Influencia } \\
\text { de otros }\end{array}$ & $\begin{array}{c}\text { Baja } \\
\text { forma }\end{array}$ & $\begin{array}{c}\text { Escasa } \\
\text { atmósfera } \\
\text { de equipo }\end{array}$ & $\begin{array}{c}\text { Edad de } \\
\text { comienzo } \\
\text { en la } \\
\text { natación } \\
\end{array}$ & $\begin{array}{c}\text { Edad de } \\
\text { finalización }\end{array}$ & $\begin{array}{l}\text { Años de } \\
\text { práctica }\end{array}$ \\
\hline & ---- & 0,189 & $0,372 * *$ & $0,316^{*}$ & $0,429 * *$ & $0,448 * *$ & $-0,145$ & $-0,370 * *$ & $-0,181$ \\
\hline \multicolumn{10}{|l|}{ Bajo rendimiento } \\
\hline Falta de diversión & & ---- & 0,089 & 0,238 & $0,388 * *$ & $0,311^{*}$ & $-0,179$ & $-0,495 * *$ & $-0,254$ \\
\hline Escasa habilidad & & & ---- & $0,396 * *$ & $0,575 * *$ & $0,307 *$ & 0,109 & $-0,310 *$ & $-0,337 *$ \\
\hline Influencia de otros & & & & ---- & $0,552 * *$ & $0,570 * *$ & $-0,048$ & $-0,403 * *$ & $-0,286^{*}$ \\
\hline Baja forma & & & & & ---- & $0,423 * *$ & $-0,172$ & $-0,487 * *$ & $-0,254$ \\
\hline $\begin{array}{c}\text { Escasa atmósfera de } \\
\text { equipo }\end{array}$ & & & & & & --- & $-0,357 *$ & $-0,478 * *$ & $-0,098$ \\
\hline $\begin{array}{c}\text { Edad de comienzo en la } \\
\text { natación }\end{array}$ & & & & & & & ---- & 0,226 & $-0,622 * *$ \\
\hline Edad de finalización & & & & & & & & ---- & $0,623 * *$ \\
\hline Años de práctica & & & & & & & & & ---- \\
\hline
\end{tabular}

En la tabla 2, se aprecia una relación positiva, entre escasa habilidad y bajo rendimiento (r: 0,372; $\mathrm{p}<0,01$ ), es decir, entre más escasa habilidad percibida mayor es la tendencia a percibir bajo rendimiento.

Por otro lado, existe una relación negativa entre años de práctica y escasa habilidad (r:-0,337; $\mathrm{p}<0,05)$, que indica que entre mayor cantidad de años de práctica menor es la percepción de escasa habilidad. Entre la relación de años de práctica e influencia de otros (r:-0,286; p<0,05), también sucede entre años de práctica y edad de comienzo ( $r:-0,622 ; p<0,01$ ), lo que indica que entre mayor cantidad de años de práctica, la edad de iniciación es menor. No existió una relación significativa entre la edad de comienzo y edad final.

Se aplicó un ANOVA de 3 vías para comparar las seis dimensiones del Cuestionario de Causas de Abandono de la Práctica Deportiva, aplicado a los sujetos, en función de su género y de su edad de inicio de práctica de la natación (antes y después de los 10 años). A continuación se presenta la estadística descriptiva de este análisis.

Tabla 3. Resumen de promedios y desviaciones estándar de las dimensiones del Cuestionario de Causas de Abandono de la Práctica Deportiva según edad de inicio de la práctica de natación y género, de ex nadadores costarricenses

\begin{tabular}{|c|c|c|c|c|c|c|c|c|}
\hline & $\begin{array}{l}\text { Inicio de la } \\
\text { práctica de } \\
\text { natación }\end{array}$ & $\begin{array}{c}\text { Bajo } \\
\text { rendimiento }\end{array}$ & $\begin{array}{c}\text { Falta de } \\
\text { diversión }\end{array}$ & $\begin{array}{c}\text { Escasa } \\
\text { habilidad }\end{array}$ & $\begin{array}{c}\text { Influencia } \\
\text { de otros }\end{array}$ & $\begin{array}{c}\text { Baja } \\
\text { forma }\end{array}$ & $\begin{array}{c}\text { Escasa } \\
\text { atmósfera } \\
\text { de equipo }\end{array}$ & $\begin{array}{c}\text { Sujetos } \\
\text { (n) }\end{array}$ \\
\hline \multirow[t]{3}{*}{ Hombres } & $\begin{array}{l}\text { Antes de los } \\
10 \text { años }\end{array}$ & $1,62 \pm 0,64$ & $3,03 \pm 1,03$ & $1,79 \pm 0,86$ & $1,66 \pm 0,79$ & $2,16 \pm 0,91$ & $2,25 \pm 1,25$ & 8 \\
\hline & $\begin{array}{l}\text { Después de } \\
\text { los } 10 \text { años }\end{array}$ & $1,68 \pm 0,76$ & $2,16 \pm 0,99$ & $2,00 \pm 0,65$ & $1,88 \pm 0,53$ & $2,18 \pm 0,57$ & $1,88 \pm 0,89$ & 9 \\
\hline & Total & $1,65 \pm 0,69$ & $2,57 \pm 1,07$ & $1,90 \pm 0,74$ & $1,78 \pm 0,65$ & $2,17 \pm 0,72$ & $2,05 \pm 1,05$ & 17 \\
\hline \multirow[t]{3}{*}{ Mujeres } & $\begin{array}{l}\text { Antes de los } \\
10 \text { años }\end{array}$ & $1,60 \pm 0,58$ & $1,81 \pm 0,89$ & $1,78 \pm 0,66$ & $1,59 \pm 0,70$ & $2,04 \pm 0,90$ & $2,39 \pm 1,31$ & 24 \\
\hline & $\begin{array}{l}\text { Después de } \\
\text { los } 10 \text { años }\end{array}$ & $1,50 \pm 0,66$ & $1,42 \pm 0,71$ & $2,01 \pm 0,44$ & $1,23 \pm 0,34$ & $1,43 \pm 0,67$ & $1,35 \pm 0,81$ & 10 \\
\hline & Total & $1,57 \pm 0,60$ & $1,69 \pm 0,85$ & $1,85 \pm 0,61$ & $1,48 \pm 0,64$ & $1,86 \pm 0,88$ & $2,08 \pm 1,27$ & 34 \\
\hline \multirow[t]{3}{*}{ Total } & $\begin{array}{l}\text { Antes de los } \\
10 \text { años }\end{array}$ & $1,61 \pm 0,59$ & $2,11 \pm 1,05$ & $1,78 \pm 0,70$ & $1,60 \pm 0,71$ & $2,07 \pm 0,89$ & $2,35 \pm 1,27$ & 32 \\
\hline & $\begin{array}{l}\text { Después de } \\
\text { los } 10 \text { años }\end{array}$ & $1,58 \pm 0,70$ & $1,77 \pm 0,91$ & $2,00 \pm 0,53$ & $1,54 \pm 0,54$ & $1,78 \pm 0,72$ & $1,60 \pm 0,87$ & 19 \\
\hline & Total & $1,60 \pm 0,62$ & $1,99 \pm 1,01$ & $1,86 \pm 0,65$ & $1,58 \pm 0,65$ & $1,97 \pm 0,83$ & $2,07 \pm 1,19$ & 51 \\
\hline
\end{tabular}




\section{MHSalud \\ URL www.una.ac.cr/mhsalud}

Tabla 4. Resumen de ANOVA de las dimensiones del Cuestionario de Causas de Abandono de la Práctica Deportiva, en función del género y edad de inicio de la práctica de la natación en ex nadadores costarricenses

\begin{tabular}{ccc}
\hline Fuente de varianza & $\mathbf{F}$ & Eta $^{\mathbf{2}}$ \\
\hline Dimensión & $4,232 * *$ & 0,083 \\
Dimensión * género & $2,952^{*}$ & 0,059 \\
Dimensión * inicio & $3,147^{* *}$ & 0,063 \\
Dimensión * género * inicio & 1,264 & 0,026 \\
Género & 4,041 & 0,079 \\
Inicio & 2,103 & 0,043 \\
Género * inicio & 0,577 & 0,012 \\
\hline
\end{tabular}

$* * \mathrm{p}<0,01 * \mathrm{p}<0,05$

En la tabla anterior, se destaca la interacción significativa de las dimensiones por género de los participantes $(\mathrm{F}: 2,952 ; \mathrm{p}<0,05)$ y la interacción de dimensiones por edad de inicio de la práctica de la natación (F: 3,147; p <0,01). En ambos casos, el Eta cuadrado es relativamente pequeño, mostrando un porcentaje de varianza explicada para ambas interacciones de aproximadamente un $6 \%$. A continuación se ilustra las interacciones mencionadas.

Gráfico 1. Interacción de dimensiones del cuestionario de causas de abandono deportivo y género en exnadadores costarricenses (n:51)

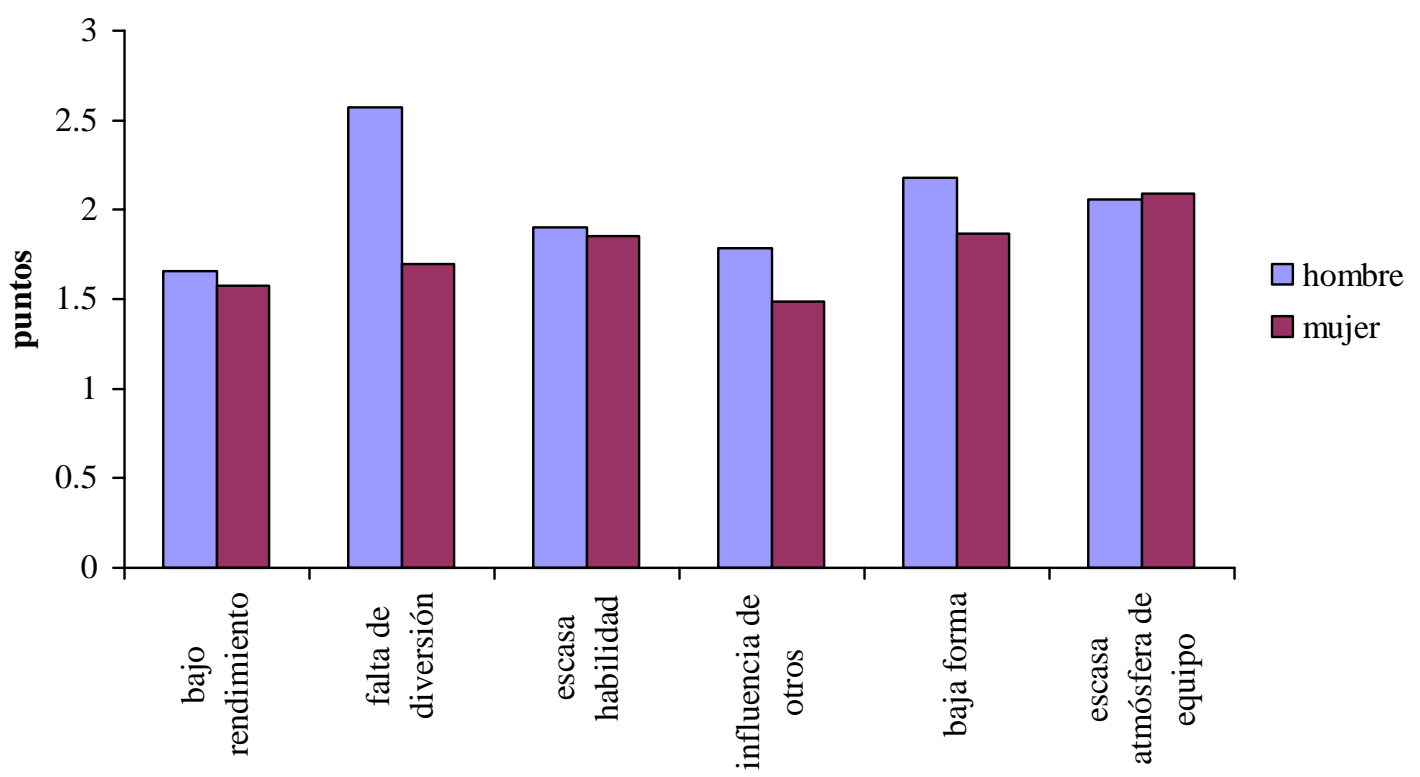

El análisis de efectos simples aplicado a la interacción de dimensiones por género demostró efecto significativo de dimensiones en los hombres así como en las mujeres, donde el análisis post-hoc mostró, en el caso de los hombres, que las dimensiones más importantes como posibles causas de abandono fueron la falta de diversión y la baja forma; sin embargo estas dimensiones no difieren significativamente de la escasa habilidad, ni de la escasa atmósfera de equipo con la cual comparten 


\section{MHSalud}

URL www.una.ac.cr/mhsalud

su importancia. En el caso de las mujeres el post-hoc demostró que la dimensión más importante como posible causa de abandono fue la escasa atmósfera de equipo y otros factores muy importantes fueron la baja forma y la escasa habilidad; por otro lado, la influencia de otros y el bajo rendimiento fueron los menos importantes. Es decir, que existe diferencia entre las posibles causas de abandono de la natación competitiva, según el género, destacando la diversión en los hombres y la atmósfera de equipo en las mujeres, pero coincidiendo ambos en la importancia de la forma física y la escasa habilidad. Se encontró efecto significativo de género en dimensiones, pero solo en cuanto a falta de diversión donde los hombres consideraron significativamente más que las mujeres, que esta dimensión podría ser importante para explicar el abandono de la práctica de la natación. Por otro lado, las otras dimensiones no mostraron diferencias entre género.

\section{Gráfico 2. Interacción de dimensiones del cuestionario de causas de abandono deportivo y edad de inicio en la natación en exnadadores costarricenses (n:51)}

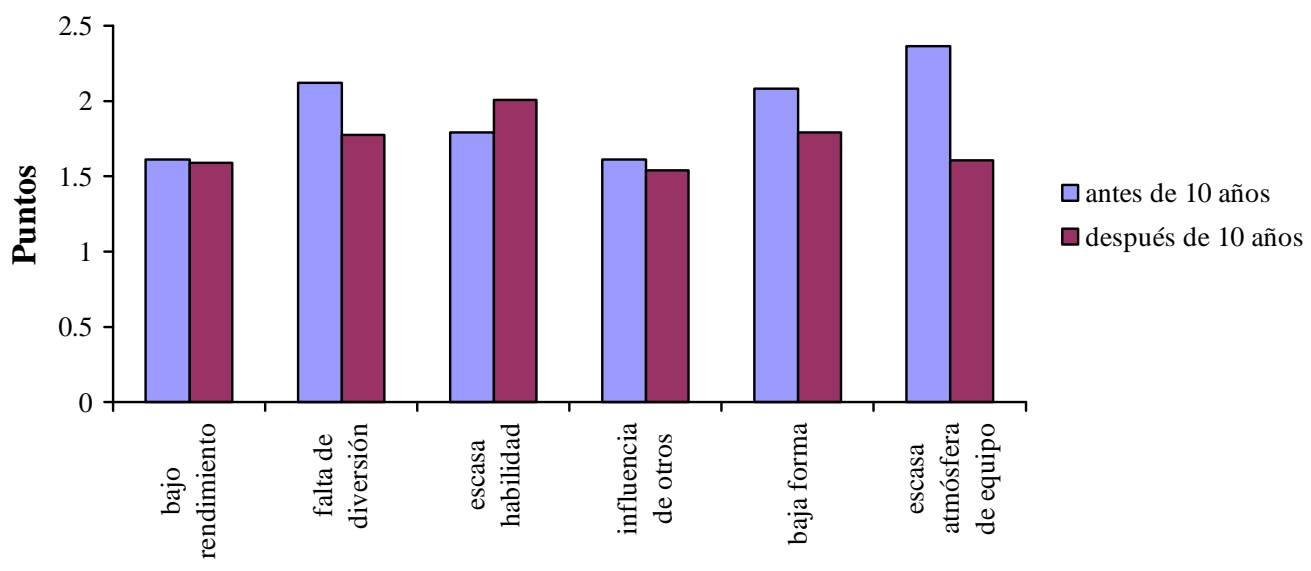

El análisis de efectos simples aplicado a la interacción, muestra efecto significativo de las dimensiones en el grupo de sujetos que inició la natación antes de los 10 años, pero no en el grupo que inició después de los diez años, lo cual indica que estos últimos sujetos valoran con una importancia similar cada una de las dimensiones del Cuestionario de Abandono Deportivo, como posibles causas de retiro de la natación competitiva. El análisis post-hoc mostró que para los sujetos que iniciaron antes de cumplir 10 años; la escasa atmósfera de equipo, la baja forma y la falta de diversión, son las posibles causas de abandono más importantes, en comparación con las demás, mientras que el bajo rendimiento y la influencia de otros son las menos importantes en estos sujetos. Cabe recordar aquí que este grupo es el que permaneció más años haciendo natación competitiva. $\mathrm{Al}$ encontrarse efecto simple significativo de la edad de inicio en la dimensión escasa atmósfera de equipo, donde los sujetos que iniciaron antes de cumplir 10 años, consideraron que esta dimensión era importante, más que los sujetos que iniciaron después de los 10 años, mientras que en las otras dimensiones no se encontraron diferencias significativas según la edad de inicio. 


\section{MHSalud}

URL www.una.ac.cr/mhsalud

Se empleó un ANOVA de 3 vías para comparar las seis dimensiones del Cuestionario de Causas de Abandono de la Práctica Deportiva, aplicado a los sujetos, en función de su género y su nivel competitivo (nacional e internacional) cuando eran nadadores. A continuación se presenta la estadística descriptiva de este análisis.

Tabla 5. Resumen de promedios y desviaciones estándar de las dimensiones del Cuestionario de Causas de Abandono de la Práctica Deportiva, según nivel competitivo (torneos nacionales o internacionales) y el género, de ex nadadores costarricenses

\begin{tabular}{|c|c|c|c|c|c|c|c|c|}
\hline & $\begin{array}{c}\text { Nivel } \\
\text { competitivo }\end{array}$ & $\begin{array}{c}\text { Bajo } \\
\text { rendimiento }\end{array}$ & $\begin{array}{c}\text { Falta de } \\
\text { diversión }\end{array}$ & $\begin{array}{c}\text { Escasa } \\
\text { habilidad }\end{array}$ & $\begin{array}{c}\text { Influencia } \\
\text { de otros }\end{array}$ & $\begin{array}{c}\text { Baja } \\
\text { forma }\end{array}$ & $\begin{array}{c}\text { Escasa } \\
\text { atmósfera } \\
\text { de equipo }\end{array}$ & $\begin{array}{c}\text { Sujetos } \\
\text { (n) }\end{array}$ \\
\hline \multirow[t]{2}{*}{ Hombres } & Nacional & $1,88 \pm 0,80$ & $2,32 \pm 1,08$ & $2,08 \pm 0,78$ & $2,03 \pm 0,69$ & $2,25 \pm 0,78$ & $2,25 \pm 1,20$ & 10 \\
\hline & Total & $1,65 \pm 0,69$ & $2,57 \pm 1,07$ & $1,90 \pm 0,74$ & $1,78 \pm 0,65$ & $2,17 \pm 0,72$ & $2,05 \pm 1,05$ & 17 \\
\hline \multirow[t]{2}{*}{ Mujeres } & Nacional & $1,60 \pm 0,71$ & $1,84 \pm 1,06$ & $2,06 \pm 075$ & $1,73 \pm 0,88$ & $2,16 \pm 1,11$ & $2,34 \pm 1,51$ & 13 \\
\hline & Internacional & $1,56 \pm 0,53$ & $1,60 \pm 0,70$ & $1,72 \pm 0,47$ & $1,33 \pm 0,37$ & $1,68 \pm 0,66$ & $1,92 \pm 1,09$ & 21 \\
\hline Total & Internacional & $1,50 \pm 0,49$ & $1,93 \pm 0,97$ & $1,70 \pm 0,51$ & $1,35 \pm 0,38$ & $1,77 \pm 0,67$ & $1,89 \pm 1,02$ & 28 \\
\hline
\end{tabular}

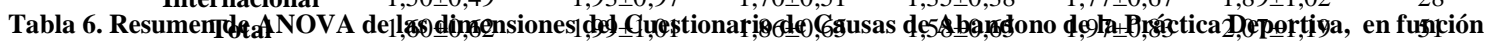
del género y del nivel competitivo en ex nadadores costarricenses

\begin{tabular}{ccc}
\hline Fuente de varianza & $\mathbf{F}$ & Eta $^{\mathbf{2}}$ \\
\hline Dimensión & $5,513^{*}$ & 0,105 \\
Dimensión * género & $3,173^{*}$ & 0,063 \\
Dimensión * nivel competitivo & 1,420 & 0,029 \\
Dimensión * género * nivel & 1,283 & 0,027 \\
Género & 1,430 & 0,030 \\
Nivel & 3,030 & 0,061 \\
$* \mathrm{p}<0,01$ & 0,021 & 0,000 \\
\hline Género * nivel & &
\end{tabular}

En la tabla anterior se observa que no hubo interacción entre las dimensiones, el nivel competitivo y el género; solo se confirma la interacción de dimensiones por género. Esto quiere decir que los sujetos califican a las dimensiones del Cuestionario de Causas de Abandono de la Práctica Deportiva con similar importancia en función de su nivel competitivo, lo cual indica que el hecho de que un nadador participara en torneos internacionales no implicaría necesariamente que vaya a ver con mayor o menor importancia algunas de las causas posibles de abandono, en comparación con sujetos que solo compitieron en torneos nacionales. Se aplicó un ANOVA de 3 vías para comparar las 6 dimensiones del Cuestionario de Causas de Abandono de la Práctica Deportiva, aplicado a los sujetos, en función de su género y su deseo de volver a practicar natación competitiva. A continuación se presenta la estadística descriptiva de este análisis.

Tabla 7. Resumen de promedios y desviaciones estándar de las dimensiones del Cuestionario de Causas de Abandono de la Práctica Deportiva, en función del género y del deseo de volver a practicar natación en ex nadadores costarricenses

\begin{tabular}{cccccccc}
\hline & $\begin{array}{c}\text { Deseo de } \\
\text { volver a } \\
\text { practicar }\end{array}$ & $\begin{array}{c}\text { Bajo } \\
\text { rendimiento }\end{array}$ & $\begin{array}{c}\text { Falta de } \\
\text { diversión }\end{array}$ & $\begin{array}{c}\text { Escasa } \\
\text { habilidad }\end{array}$ & $\begin{array}{c}\text { Influencia de } \\
\text { otros }\end{array}$ & $\begin{array}{c}\text { Baja forma } \\
\text { Escasa } \\
\text { atmósfera } \\
\text { de equipo }\end{array}$ & $\begin{array}{c}\text { Sujetos } \\
\text { (n) }\end{array}$ \\
\hline Hombres & $\mathbf{S i}$ & $1,73 \pm 0,80$ & $2,39 \pm 1,00$ & $1,91 \pm, 077$ & $1,90 \pm 0,70$ & $2,22 \pm 0,74$ & $2,33 \pm 1,11$ \\
& No & $1,48 \pm 0,26$ & $3,00 \pm 1,23$ & $1,86 \pm 0,73$ & $1,50 \pm 0,45$ & $2,06 \pm 0,75$ & $1,40 \pm 0,54$ \\
Mujeres & Total & $1,65 \pm 0,69$ & $2,57 \pm 1,07$ & $1,90 \pm 0,74$ & $1,78 \pm 0,65$ & $2,17 \pm 0,72$ & $2,05 \pm 1,05$ \\
& Si & $1,55 \pm 0,61$ & $1,67 \pm 0,84$ & $1,86 \pm 0,62$ & $1,53 \pm 0,66$ & $1,88 \pm 0,89$ & $2,16 \pm 1,32$ \\
& No & $1,75 \pm 0,55$ & $1,87 \pm 1,01$ & $1,75 \pm 0,61$ & $1,12 \pm 0,15$ & $1,70 \pm 0,90$ & $1,50 \pm 0,57$ \\
Total & Total & $1,57 \pm 0,60$ & $1,69 \pm 0,85$ & $1,85 \pm 0,61$ & $1,48 \pm 0,64$ & $1,86 \pm 0,88$ & $2,08 \pm 1,27$ \\
& Si & $1,60 \pm 0,66$ & $1,88 \pm 0,94$ & $1,88 \pm 0,66$ & $1,63 \pm 0,68$ & $1,98 \pm 0,85$ & $2,21 \pm 1,25$ \\
& No & $1,60 \pm 0,41$ & $2,50 \pm 1,22$ & $1,81 \pm 0,64$ & $1,33 \pm 0,39$ & $1,90 \pm 0,79$ & $1,44 \pm 0,52$ \\
& Total & $1,60 \pm 0,62$ & $1,99 \pm 1,01$ & $1,86 \pm 0,65$ & $1,58 \pm 0,65$ & $1,97 \pm 0,83$ & $2,07 \pm 1,19$ \\
\hline
\end{tabular}




\section{MHSalud}

URL www.una.ac.cr/mhsalud

Tabla 8. Resumen de ANOVA de las dimensiones del Cuestionario de Causas de Abandono de la Práctica Deportiva, en función del género y del deseo de volver a practicar natación en ex nadadores costarricenses

\begin{tabular}{ccc}
\hline Fuente de varianza & F & Eta $^{\mathbf{2}}$ \\
\hline Dimensión & $4,000^{* *}$ & 0,078 \\
Dimensión * género & 1,945 & 0,040 \\
Dimensión * volver & $2,485^{*}$ & 0,050 \\
Dimensión * género * volver &, 335 & 0,007 \\
Género & 1,734 & 0,036 \\
Volver &, 694 & 0,015 \\
Género * volver &, 007 & 0,000 \\
\hline
\end{tabular}

$* * \mathrm{p}<0,01 * \mathrm{p}<0,05$

Se encontró interacción significativa de dimensiones y deseo de volver a practicar la natación. El análisis de efectos simples halló efectos significativos de las dimensiones en el grupo de sujetos que deseaban volver a nadar así como en aquellos que no lo desean.

Gráfico 3. Interacción de dimensiones del cuestionario de causas de abandono deportivo y deseo de volver a practicar la natación en exnadadores costarricenses (n:51)

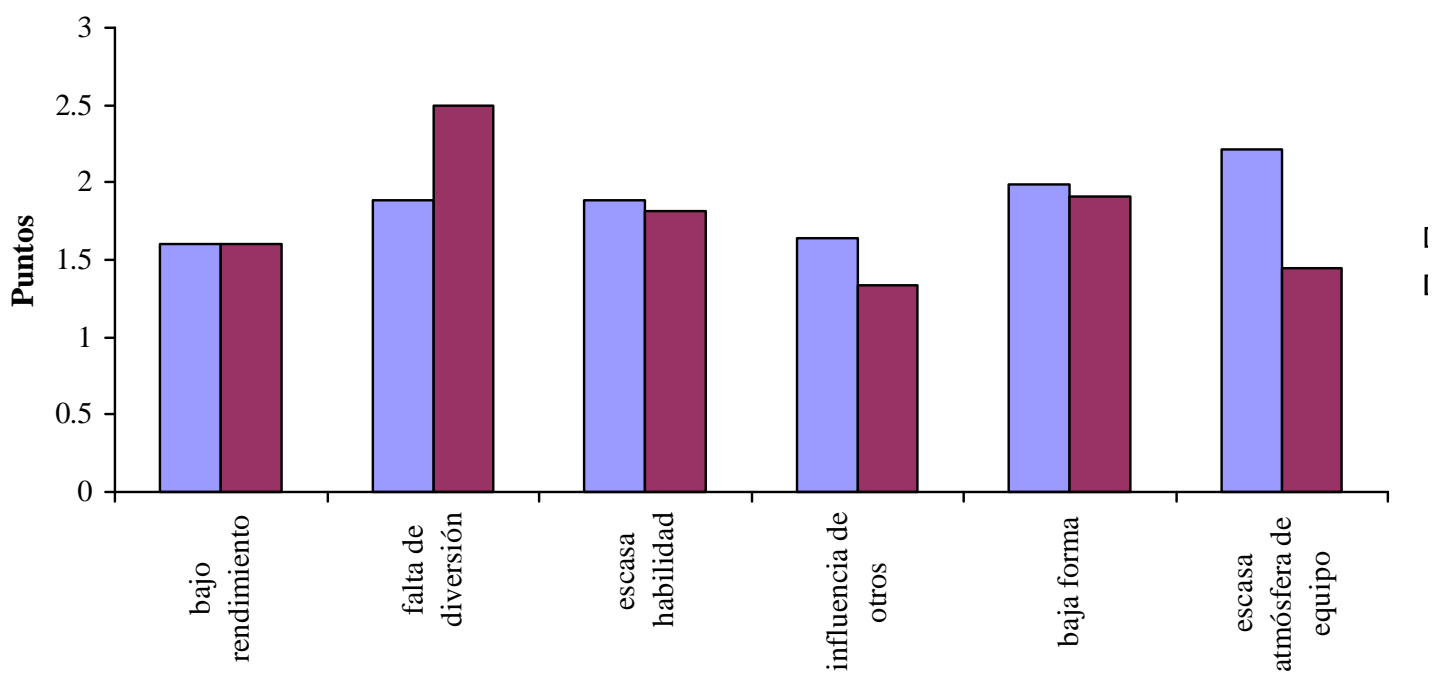

El análisis post-hoc en los sujetos que sí deseaban volver a nadar, mostró que la escasa atmósfera de equipo, la baja forma y la escasa habilidad fueron las dimensiones más importantes como posibles causas de abandono, mientras que el bajo rendimiento y la influencia de otros fueron las menos importantes. En el caso de los sujetos que no desean volver a nadar, el post-hoc expuso que la falta de diversión fue más importante que la influencia de otros y la escasa atmósfera de equipo, como posible causa de abandono, lo cual muestra que hay diferencias entre ambos grupos de sujetos con respecto a la importancia que se le otorga a una dimensión o a otra como posible causa de abandono.

Tabla 9. Resumen de promedios y desviaciones estándar de la edad de inicio de la práctica de la natación (antes de los diez años, después de los diez años) en ex nadadores costarricenses

\begin{tabular}{|c|c|c|c|}
\hline Género & Edad de inicio & & $\mathbf{N}$ \\
\hline \multirow[t]{3}{*}{ Hombre } & Antes de 10 años & $8,50 \pm 3,20$ & 8 \\
\hline & Después de 10 años & $4,66 \pm 1,87$ & 9 \\
\hline & Total & $6,47 \pm 3,18$ & 17 \\
\hline \multirow[t]{2}{*}{ Mujer } & 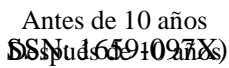 & $9,00 \pm 3,38$ & $\begin{array}{l}24 \\
10\end{array}$ \\
\hline & Total & $7,67 \pm 3,67$ & 34 \\
\hline \multirow[t]{3}{*}{ Total } & Antes de 10 años & $8,87 \pm 3,29$ & 32 \\
\hline & Después de 10 años & $4,57 \pm 1,92$ & 19 \\
\hline & Total & $7,27 \pm 3,53$ & 51 \\
\hline
\end{tabular}




\section{MHSalud}

URL www.una.ac.cr/mhsalud

Tabla 10. Resumen de ANOVA en función del género y del inicio de la práctica de la natación en ex nadadores costarricenses

$* \mathrm{p}<0,05$

\begin{tabular}{ccc}
\hline Fuente de varianza & $\mathbf{F}$ & $\mathbf{E t a}^{\mathbf{2}}$ \\
\hline Género & 0,034 & 0,001 \\
Inicio & $21,465^{*}$ & 0,314 \\
Género * inicio & 0,137 & 0,003 \\
\hline
\end{tabular}

En la tabla anterior se observa que existieron diferencias significativas en los años de práctica en los sujetos según su edad de inicio en este deporte, donde los sujetos que iniciaron antes de 10 años, tuvieron un promedio de años de práctica significativamente más alto que los sujetos que empezaron después de 10 años. No se encontró diferencias según el género; los varones (6,47 años) y las mujeres (7,67 años) tuvieron años de práctica promedio similares.

Tabla 11. Resumen de promedios y desviaciones estándar del nivel competitivo (Internacional o nacional) alcanzado por ex nadadores costarricenses

\begin{tabular}{|c|c|c|c|c|c|}
\hline Género & Nivel competitivo & & & $\mathbf{n}$ & \\
\hline \multirow[t]{3}{*}{ Hombre } & Nacional & $6,00 \pm 2,98$ & & 10 & \\
\hline & Internacional & $7,14 \pm 3,57$ & & 7 & \\
\hline & Total & $6,47 \pm 3,18$ & & 17 & \\
\hline \multirow[t]{3}{*}{ Mujer } & Nacional & $6,46 \pm 3,92$ & & 13 & \\
\hline & Internacional & $8,42 \pm 3,38$ & & 21 & \\
\hline & Total & $7,67 \pm 3,67$ & & 34 & \\
\hline Total & Nacional & $6,26 \pm 3,48$ & & 23 & \\
\hline Género & & $\mathrm{Si}$ & No & & Total \\
\hline $\begin{array}{c}\text { Hombres } \\
\text { Muieres }\end{array}$ & $\begin{array}{l}\text { Hecional } \\
\text { Fetal } \\
\text { Tetalduencia }\end{array}$ & $\begin{array}{l}8,10 \pm \frac{124}{4} \\
7,27 \pm 30 \\
53\end{array}$ & $\begin{array}{l}5 \\
4\end{array}$ & $\begin{array}{l}28 \\
51\end{array}$ & $\begin{array}{l}17 \\
34\end{array}$ \\
\hline
\end{tabular}

Tabla 12. Resumen de ANOVA en función del género y del nivel competitivo de la práctica de la natación en ex nadadores costarricenses

\begin{tabular}{ccc}
\hline Fuente de varianza & F & Eta $^{\mathbf{2}}$ \\
\hline Género & 0,684 & 0,014 \\
Nivel competitivo & 2,166 & 0,044 \\
Género * nivel competitivo & 0,152 & 0,003 \\
\hline
\end{tabular}

No se encontró diferencias en la cantidad de años de práctica de la natación competitiva según el género ni el nivel competitivo de los ex nadadores entrevistados.

Tabla 13. Frecuencia de respuesta al deseo de volver a practicar el deporte de la natación

Se realizó una $\mathrm{Chi}^{2}$ para analizar la respuesta entre hombres y mujeres y no se encontró diferencias en la respuesta por hombres o mujeres (X: 0,$119 ; \mathrm{p}>0,05)$. 


\section{MHSalud \\ URL www.una.ac.cr/mhsalud}

Tabla 14. Resumen de ANOVA en función del género y del deseo de volver a practicar de la natación en ex nadadores costarricenses

\begin{tabular}{ccc}
\hline Fuente de varianza & F & Eta $^{\mathbf{2}}$ \\
\hline Género & 0,713 & 0,015 \\
Deseo de volver & 0,041 & 0,001 \\
Género * deseo de volver & 0,020 & 0,000
\end{tabular}

No existió diferencia en los años de práctica de la materia competitiva según el género, ni el deseo de practicar otra vez la natación. Es decir, que el deseo de volverlo hacer en los ex nadadores entrevistados, no está en función de la cantidad de años que estos sujetos estuvieron nadando.

Tabla 15. Resumen de promedios y desviaciones estándar de las dimensiones del Cuestionario de Causas de Abandono de la Práctica Deportiva, en función de las horas de práctica de la natación en ex nadadores costarricenses

\begin{tabular}{|c|c|c|c|c|c|c|c|}
\hline $\begin{array}{l}\text { Horas de práctica de } \\
\text { natación }\end{array}$ & $\begin{array}{c}\text { Bajo } \\
\text { rendimiento }\end{array}$ & $\begin{array}{c}\text { Falta de } \\
\text { diversión }\end{array}$ & $\begin{array}{c}\text { Escasa } \\
\text { habilidad }\end{array}$ & $\begin{array}{l}\text { Influencia } \\
\text { de otros }\end{array}$ & $\begin{array}{c}\text { Baja } \\
\text { forma }\end{array}$ & $\begin{array}{c}\text { Escasa } \\
\text { atmósfera } \\
\text { de equipo }\end{array}$ & $\begin{array}{l}\text { Sujetos } \\
\text { (n) }\end{array}$ \\
\hline $\begin{array}{c}\text { Menos de } 6 \text { horas a } \\
\text { la semana }\end{array}$ & $1,55 \pm 0,80$ & $1,43 \pm 0,85$ & $2,29 \pm 0,77$ & $1,60 \pm 0,90$ & $2,06 \pm 1,42$ & $2,31 \pm 1,57$ & 8 \\
\hline $\begin{array}{c}\text { De } 6 \text { a } 12 \text { horas a la } \\
\text { semana }\end{array}$ & $1,81 \pm 0,64$ & $2,73 \pm 0,99$ & $1,94 \pm 0,58$ & $1,46 \pm 0,30$ & $2,16 \pm 0,72$ & $2,03 \pm 1,14$ & 15 \\
\hline $\begin{array}{c}\text { De } 12 \text { a } 15 \text { horas a la } \\
\text { semana }\end{array}$ & $1,65 \pm 0,66$ & $1,95 \pm 1,01$ & $1,72 \pm, 65$ & $1,73 \pm 0,87$ & $1,88 \pm 0,76$ & $2,26 \pm 1,27$ & 15 \\
\hline Más de 15 horas & $1,33 \pm 0,34$ & $1,51 \pm 0,58$ & $1,69 \pm 0,58$ & $1,53 \pm 0,49$ & $1,78 \pm 0,59$ & $1,76 \pm 0,92$ & 13 \\
\hline Total & $1,60 \pm 0,62$ & $1,99 \pm 1,01$ & $1,86 \pm 0,65$ & $1,58 \pm 0,65$ & $1,97 \pm 0,83$ & $2,07 \pm 1,19$ & 51 \\
\hline
\end{tabular}

Tabla 16. Resumen de ANOVA en función de las dimensiones del Cuestionario de Causas de Abandono de la Práctica Deportiva y las horas de práctica de natación en ex nadadores costarricenses

\begin{tabular}{ccc}
\hline Fuente de varianza & F & Eta $^{\mathbf{2}}$ \\
\hline Dimensiones & 4,673 & 0,090 \\
Dimensiones * horas de práctica & $2,072^{*}$ & 0,117 \\
Horas de práctica & 1,272 & 0,075 \\
\hline
\end{tabular}

$* \mathrm{p}<0,05$

Se encontró interacción significativa de dimensiones por horas de práctica semanal de natación, cuando los sujetos eran nadadores activos, esto se ilustra en el siguiente gráfico.

Gráfico 4. Interacción de dimensiones del cuestionario de causas de abandono deportivo y horas de práctica de natación en exnadadores costarricenses (n:51)

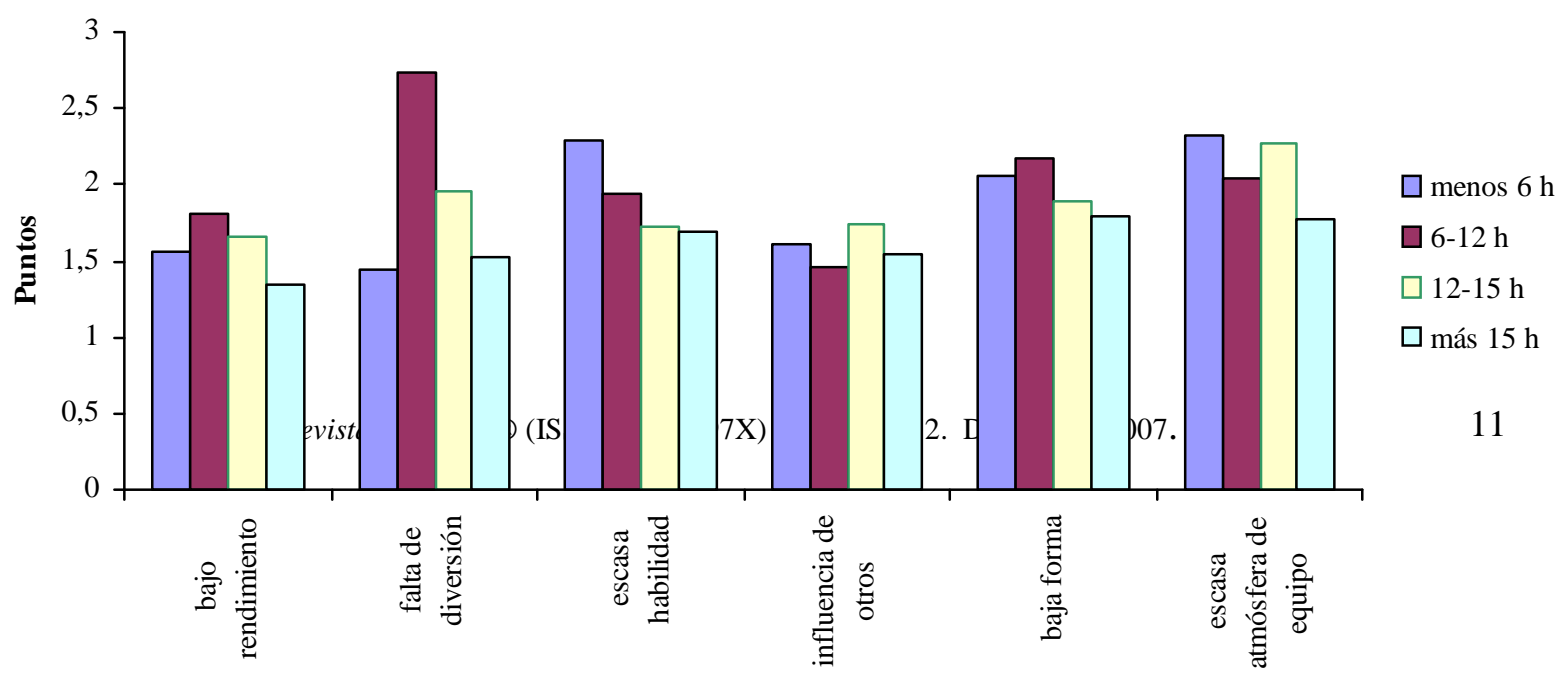




\section{MHSalud}

URL www.una.ac.cr/mhsalud

El análisis de Efectos Simples muestra un efecto significativo de dimensiones en el grupo de sujetos que nadaban menos de seis horas. El análisis post hoc mostró que para estos sujetos la dimensión "Escasa Atmósfera de equipo" es más importante que la "Bajo rendimiento" y "Falta de diversión", y que la "Escasa habilidad" es más importante que la "Bajo Rendimiento", "Falta de diversión" e "Influencia de otros". La "Bajo rendimiento" menos importante que la "Escasa habilidad" y "Escasa atmósfera de equipo". La "Falta de diversión" menos importante que la "Escasa habilidad" y "Escasa atmósfera de equipo". Por otro lado, la "Influencia de otros" menos importante que la "Escasa habilidad". También hubo efecto simple significativo en los sujetos que nadaban de 6 a 12 horas. El análisis post-hoc mostró que la dimensión "Falta de diversión" fue más importante que la "Bajo rendimiento", "Escasa habilidad" e "Influencia de otros"; y la "Baja forma" más importante que la "Influencia de otros". Además no se encontró efecto simple significativo de dimensiones en el grupo de sujetos que nadaba de 12 a 15 horas y tampoco sucedió así en el grupo que nadaba más de 15 horas, lo cual indica que estos dos últimos grupos de sujetos vieron con igual importancia a las seis dimensiones del Cuestionario de Causas de Abandono. Por otro lado se encontró efecto simple significativo de las horas de práctica semanal en la dimensión "Falta de diversión". El posthoc de Turkey mostró que la "Falta de diversión" era la más importante como posible causa de abandono paras los sujetos que nadaban de seis a doce horas en comparación a los nadadores que practicaban menos de seis horas y en comparación con los que lo hacían más de 15 horas semanales. En las otras dimensiones no se encontró efecto significativo en las horas de práctica.

Tabla 17. Resumen de promedios y desviaciones estándar de las horas de práctica de ex nadadores costarricenses

\begin{tabular}{ccc}
\hline Horas de práctica & $\mathbf{N}$ \\
\hline Menos de 6 horas a la semana & $5,62 \pm 3,81$ & 8 \\
De 6 a 12 horas a la semana & $6,93 \pm 3,47$ & 15 \\
De 12 a 15 horas a la semana & $6,66 \pm 3,10$ & 15 \\
Más de 15 horas & $9,38 \pm 3,30$ & 13 \\
Total & $7,27 \pm 3,53$ & 51 \\
\hline
\end{tabular}

Se aplicó un Análisis de Varianza de una Vía para determina si existía diferencia en los años de práctica de la natación competitiva que tuvieron los sujetos según la cantidad de horas de entrenamiento semanal que tenían los sujetos siento nadadores activos. El ANOVA mostró que no hubo diferencias significativas (F: 2,535; $\mathrm{p}>0,05)$, es decir que para los sujetos entrevistados en este estudio la cantidad de años que se mantuvieron compitiendo en natación es independiente de la cantidad de horas de entrenamiento semanal, por lo cual se podría afirmar que existen otras razones con mayor peso para explicar el retiro en estos sujetos. 


\section{MHSalud}

URL www.una.ac.cr/mhsalud

\section{DISCUSIÓN}

El objetivo de esta investigación fue determinar la importancia de distintos factores para explicar el abandono de la práctica deportiva de la natación en hombres y mujeres costarricenses y según la edad a la que se retiraron.

Según Águila y Andujar (2000), la edad de comienzo no se relaciona con la de abandono, lo que sugiere que la especialización a temprana edad no es perjudicial, mientras se respete los liniamientos de entrenamiento para estas ellas. El estudio refleja que los nadadores, se retiran a edades tempranas antes de alcanzar el rendimiento máximos, lo que podría indicar que esos límites de especialización temprana se están violentando.

Por otro lado, los nadadores que perciben escasa habilidad, tienden a recibir bajo rendimiento en la práctica de la natación, esto debido a que los individuos valoran los resultados en proporción con sus expectativas de lograrlo, de forma que el nadador que perciba poca habilidad verá menos posibilidades de alcanzar su meta y con ello el abandono (Schunk, 1997 y Weinberg y Gould, 1996) por otro lado un nadador que percibe habilidad sentirá más posibilidades de alcanzar su metas, así podrá mantener la motivación durante más años. Estos resultados coinciden con los hallazgos de Martín y Dodder (1991).

Otro hallazgo es que aquellos nadadores con mayor cantidad de años de práctica perciben la influencia de otros con menos importancia (amigos y padres de familia como motivación extrínsecos). Esto podría deberse al tipo de motivación que reine en el sujeto, la intrínseca provoca una determinación propia en los deportistas y en su actividad, proporciona una motivación duradera, siendo esta interna, poco influirán otras personas, además la conducta extrínseca, es motivada por la posibilidad de adquirir algún objeto o reconocimiento, de esta forma si se observa que en la natación costarricense no existen grandes premios ni gran prestigio social se estaría reforzando la idea que la motivación reinante es la intrínseca (Greco y Gorostarzu, 2002).

Otro resultado fue que la edad de comienzo de la práctica es menor, la cantidad de años de práctica va a ser mayor. Se debe recordar que la edad media de abandono es entre los 16 años y los 19 años, donde la mayoría de personas se encuentran en el último año de colegio y cerca de ingresar a las carreras universitarias, esto lo reitera en un estudio Ruiz y García (2002), donde demuestran que el abandonado de la práctica deportiva corresponde frecuentemente a las poblaciones próximas a épocas de cambios de estudios obligatorios, o al mundo laboral o al familiar.

De acuerdo al estudio los hombres consideran las dimensiones más destacadas como causas de abandono, la falta de diversión y la baja forma, sin embargo, estas no difieren significativamente de la escasa habilidad, ni de la poca atmósfera de equipo. Por otro lado, las mujeres mostraron que las dimensiones más importantes como posibles causas de abandono fueron la escasa atmósfera de equipo, la baja forma y la poca habilidad. Es decir, los hombres destacan la diversión y las mujeres la atmósfera de equipo, pero coincidiendo ambos en la importancia de la baja forma y la escasa habilidad. (Weinberg y Gould, 1996, Águila y Andujar, 2000 y Valdés, 1996). 


\section{MHSalud}

URL www.una.ac.cr/mhsalud

Referido a la diferencia entre género se puede especular que por lo general los hombres obtienen mayor libertad social a una menor edad, además de tener mayor acceso a otros deportes más populares donde encuentran otro tipo de diversiones más atrayentes además de ampliar su círculo de amistades naturales contribuye a satisfacer la necesidades de filiación y disociarse del deporte que no brinda o restringe estas libertades. Mientras que la mujer, no cuenta con estas ventajas hasta una edad más tardía, lo que podría dejarle como única alternativa de diversión la práctica deportiva, por lo que busca vínculos de amistad y diversión dentro del equipo, tomando mayor importancia la atmósfera de este y al ser la natación un deporte donde no se puede hablar, ni proporciona una gran cohesión de grupo, esto podría generar insatisfacción de las necesidades de filiación y aumenta las probabilidades de abandonar (Weinberg y Gould, 1996).

Los nadadores del estudio que sí deseaban volver a nadar, valoran la escasa atmósfera de equipo, la baja forma y la poca habilidad como las dimensiones más importantes para explicar el abandono de la natación. En el caso de los sujetos que no desean volver a nadar, la falta de diversión, la influencia de otros y la escasa atmósfera de equipo, fueron las principales causas de abandono, se pudo observar claras diferencias entre ambos grupos. Esto se podría explicar ya que aquel grupo que sí desea volver ha llenado sus necesidades en otros lugares, con lo que el hecho de volver a practicar deporte se ve positivamente. Para el otro grupo, se puede pensar que no tuvieron la suficiente motivación intrínseca para seguir pues ya no era divertido ni les causaba satisfacción entonces no existe motivos para reintegrarse (Weinberg y Gould, 1996, 2003, Año, 1997 y Schunk, 1997).

En el grupo que nadaban menos de seis horas demuestran que la dimensión escasa habilidad es la más importante, junto con la baja forma y poca atmósfera de equipo y esto se puede explicar al decir que hay una relación negativa entre la percepción de escasa habilidad, poco rendimiento y se percibe una tarea elevada con mínima recompensa, causa desmotivación (Schunk, 1997 y Weinberg y Gould, 1996). Por otro lado el menor contacto con sus compañeros puede explicar la percepción de baja atmósfera de equipo (Weinberg y Gould, 1996 y Año, 1997). Por otro lado se puede presumir que los sujetos del estudio tienen una motivación intrínseca, esto puede explicar la poca influencia que tienen los demás (Weinberg y Gould, 1996 y Año, 1997).

En los sujetos que nadaban de 6 a 12 horas mostró que la dimensión falta de diversión fue la más importante seguida de la baja forma y la atmósfera de equipo y menos importante la escasa habilidad y el bajo rendimiento, se puede reafirmar que el hecho de no divertirse viene a afirmar la posible ausencia de una motivación intrínseca y si a eso se le suma que la tarea es percibida como difícil caerá en la desmotivación y esto puede llevar a una atmósfera negativa en el deporte con lo que afecta su relación en la atmósfera de equipo. (Weinberg y Gould, 1996, Año, 1997; Weinberg y Gould, 1996 y Schunk, 1997).

Para los sujetos entrevistados en este estudio los resultados concluyeron que la cantidad de años que se mantuvieron compitiendo en natación es independiente de la cantidad de horas de entrenamiento semanal, aunque al preguntar las horas de práctica no se tomó en cuenta que se realizaba en esas horas, en este sentido existen teorías que afirman que 2 horas de entrenamiento diarios son más que suficientes para alcanzar niveles de preparación de élite, y previendo el sobre entrenamiento (Costill, Maglischo y Richardson, 1994 y Aldo, 1998), pero se debe explorar más en que consisten esas horas de entrenamiento cuanto de ese tiempo se dedican a nadar o a otras actividades. 


\section{MHSalud \\ URL www.una.ac.cr/mhsalud}

Se observó que la mayor parte de los sujetos $(72.5 \%)$, practicaban la natación tanto por entrenar como por competir, mientras que un $17.6 \%$ lo hacía por solo competir y un $9.8 \%$ lo hacía solo por entrenar, lo que reafirma el hecho que muy pocos nadadores tenían una motivación extrínseca (Schunk, 1997 y Weinberg y Gould, 1996).

\section{CONCLUSIONES}

La principal causa de abandono fue la falta de diversión para los hombres, mientras que para las mujeres fue la escasa atmósfera de equipo. Pero la mayoría de sujetos tiene el deseo de volver a practicar el deporte.

Se concluye que a la hora de abandonarlo, la mayoría de nadadores se encontraban estudiando, la mayor deserción se dio mientras estaban en secundaria y si se observa las edades de abandono, estas coinciden con la cercanía del cambio de colegio a la universidad

Los sujetos que nadaban menos de seis horas perciben con mayor importancia la escasa habilidad, en los que lo hacían de 6 a 12 horas una falta de diversión, para los sujetos que nadaban de 12 a 15 horas y el grupo que nadaba más de 15 horas percibieron de de igual forma todas las dimensiones del cuestionario.

Por otro lado, el hecho de que un nadador participara en torneos internacionales o nacionales no implicaría necesariamente que vaya a ver con mayor o menor importancia algunas de las causas posibles de abandono.

Los sujetos del grupo que iniciaron después de los diez años, valoran con una importancia similar las dimensiones, como posibles causas de retiro, mientras que el grupo que inició la natación antes de los 10 años, la escasa atmósfera de equipo, la baja forma y la falta de diversión, son las posibles causas de abandono más importantes.

Los sujetos que iniciaron antes de los 10 años a nadar tienden a tener un promedio de años de práctica más alto que los que empezaron después esta edad, pero entre mayor cantidad de años de práctica, menor es la percepción de escasa habilidad, y menos influencia de otros.

En los sujetos que sí deseaban volver a nadar, mostró que la escasa atmósfera de equipo, la baja forma y la poca habilidad fueron las dimensiones más importantes como posibles causas de abandono. En el caso de los sujetos que no desean volver a nadar, la falta de diversión fue la causa más importante.

Se puede concluir que entre más escasa habilidad percibida por los sujetos mayor es la tendencia a percibir bajo rendimiento en la práctica de la natación.

Por otro lado, entre mayor cantidad de años de práctica, menor es la percepción de escasa habilidad, menor percepción de influencia de otros y es menor la edad de comienzo.

\section{REFERENCIAS}

Año, V. (1997). Planificación y organización del entrenamiento juvenil. Madrid, España: Editorial Gymnos. 


\section{MHSalud}

URL www.una.ac.cr/mhsalud

Águila, C. y Andujar, C. (2000). Reflexiones acerca del entrenamiento en la infancia y la selección de talentos deportivos. Fuente: http://www.efdeportes.com. Accesada el 18 junio del 2007.

Aldo, N. (1998). Natación: entrenamiento de base en los niños y jóvenes. Fuente: http://www.efdeportes.com. Accesada el 13 junio del 2007.

Cohen, R. (1998). El Síndrome de Sobreentrenamiento. Fuente: http://www.efdeportes.com. Accesada el 25 de junio del 2006.

Costill, D., Maglischo, E. y Richardson, A. (1994). Natación aspectos biológicos y mecánicos, técnica y entrenamiento, test, controles y aspectos médicos. Barcelona, España: Editorial Hispano Europea S.A.

Cruz, J. (1997). Psicología del deporte, personalidad evaluación y tratamiento. Madrid, España: Editorial Síntesis S.A.

García, F. (2001). El Entrenador Deportivo, Cuba. [Artículo on-line] http://hsra2.com.ar/entrena/metodos/psico.html. Accesada el 28 de agosto del 2005.

Gould, D., Feltz, D. y Weiss, M. (1985). Motives for participating in competitive youth swimming. International Journal of Sport Psycology. 16, 126-140.

Greco, V. y Gorostarzu, J. (2002). Motivación en el deporte y su influencia en los resultados laborales.

[Artículo on-line] www.cema.edu.ar/postgrado/download/tesinas2002/Grecco_MADE.pdf+motivacion+depor tes\&hl=es\&ie=UTF-8. Accesada el 30 de agosto del 2003.

Martín, D.E. y Dodder, R.A. (1991). Socialization experiences and level of terminating sport participation. J Sport Behav. 14, 1134-128.

Nunnally, J.C. (1978). Psychometric Theory. New York: McGrawHill.

Ruiz, J. y García, M. (2002). El abandono y la ausencia de práctica de actividad físico-deportiva de tiempo libre del alumnado de la universidad de Almería. Un estudio longitudinal. Fuente: http://www.efdeportes.com. Accesada el 25 de agosto del 2003.

Salguero, A., Tuero, C. y Márquez, S. (2003). Adaptación española del cuestionario de causas de abandono en la práctica deportiva: validación y diferencias de género en jóvenes nadadores. Fuente: http://www.efdeportes.com. Accesada el 25 de agosto del 2003.

Schunk, D. (1997). Teorías del aprendizaje. Segunda Edición. Juárez, México: Prentice-Hall Hispanoamericana, S.A

Trepode, N. (2001). Abandono del deporte en los jóvenes. Fuente: http://www.efdeportes.com. Accesada el 23 de agosto del 2006. 


\section{MHSalud}

URL www.una.ac.cr/mhsalud

Valdés, H. (1996). La preparación psicológica del deportista: mente y rendimiento. España: Editorial: INDE.

Vasalo, C. (2001). Deporte: Un trabajo de Alto Riesgo. PubliCE [artículo on-line] (http://www.sobreentrenamiento.com/PubliCE/Home.asp). Accesada el 23 de agosto del 2005.

Weinberg, R. y Gould, D. (1996). Fundamentos de Psicología del Deporte y el Ejercicio Físico. España: Editorial Ariel, S. A.

Willmore, J. y Costill, D. (2000). Fisiología del esfuerzo y del deporte. Tercera Edición. Barcelona, España: Editorial Paidotribo.

Fecha de recepción del artículo: 03 de mayo del 2007.

Fecha de aceptación del artículo: 19 de setiembre del 2007.

Fecha de publicación del artículo: 31 de diciembre del 2007. 